\title{
Goal-oriented inverse analysis - application to building thermal problems
}

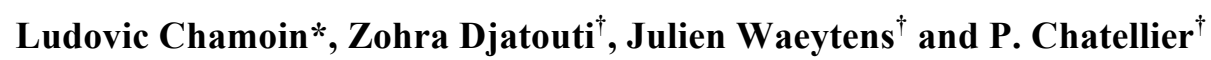 \\ * Université Paris-Saclay, ENS Paris-Saclay, CNRS, LMT \\ 4 Avenue des Sciences, 91190 Gif-Sur-Yvette, France \\ Email: ludovic.chamoin@ens-paris-saclay.fr \\ ${ }^{\dagger}$ Université Gustave Eiffel \\ 14-20 Boulevard Newton, 77447 Marne-La-Vallée, France \\ Email: \{zohra.djatouti,julien.waeytens,patrice.chatellier\}@univ-eiffel.fr
}

\begin{abstract}
We present an original goal-oriented inverse method for model parameter identification. Initially developed in [1], it is based on dual analysis and consists in a modified version of the concept of Constitutive Relation Error. It stands out from standard deterministic inverse methods by its formulation, as it focuses on the accurate prediction of a specific quantity of interest, and aims at automatically identifying and updating the model parameters involved in its computation alone.

To further reduce the CPU time of the multi-query inversion process, that also involves coupled time forward/backward systems to solve, the goal-oriented inverse method is beneficially complemented with model order reduction, using the Proper Generalized Decomposition (PGD) technique [2].

Eventually, the goal-oriented method is also employed for optimal sensor placement (experimental design), in order to drastically reduce the number of sensors which need to be deployed for the objective of the simulation.

The proposed method is here applied and illustrated with transient heat transfer models encountered in building thermal problems. Inverse analysis with such models is a compulsory but costly task for the reliable evaluation of building thermal properties, accurate prediction of energy consumption, and therefore optimal driving of renovation strategy of power control. It usually involves a large number of parameters but a restricted number of available sensing data.

In this framework, the goal-oriented method is first integrated in a software chain containing CAD and thermal dynamic simulation tools. Performance is then numerically evaluated on several test cases with real experiments. In particular, a two-story building of the Sense-City equipment with controlled climate scenarios and in-situ sensors is considered, with various quantities of interest [3]. It is shown that for all these and using the goal-oriented strategy, very few model parameters actually need to be updated, and little sensing information is necessary, without sacrificing accuracy. It is then a tool of choice for such applications.
\end{abstract}

\section{REFERENCES}

[1] L. Chamoin, J. Waeytens and P. Ladevèze, "Goal-oriented updating of mechanical models using the adjoint framework", Computational Mechanics, Vol. 54(6), pp. 1415-1430 (2014).

[2] Z. Djatouti, J. Waeytens, L. Chamoin and P. Chatellier, "Coupling a goal-oriented inverse method and Proper Generalized Decomposition for fast and robust prediction of quantities of interest in building thermal problems", Building Simulation, Vol. 13, pp. 709-727 (2020).

[3] Z. Djatouti, J. Waeytens, L. Chamoin and P. Chatellier, "Goal-oriented sensor placement and model updating strategies applied to a real building in the Sense-City equipment under controlled winter and heat wave scenarios", Energy and Buildings, in press (2020). 